\title{
Nonlinear Tempering of Subdiffusion with Chemotaxis, Volume Filling, and Adhesion
}

\author{
S. Falconer ${ }^{1 *}$, A. Al-Sabbagh ${ }^{2}$, S. Fedotov ${ }^{1}$ \\ ${ }^{1}$ School of Mathematics, The University of Manchester, Manchester M13 9PL, United Kingdom \\ ${ }^{2}$ Department of Mathematics, Al-Nahrain University, Jadiriya 64055, Baghdad, Iraq
}

\begin{abstract}
The purpose of this paper is to implement nonlinear particle interactions into subdiffusive transport, involving chemotaxis and nonlinear effects such as volume filling and adhesion. We systematically derive nonlinear subdiffusive fractional equations with chemoattractant dependent forcing. We consider the diffusion limit of the master equation and analyse the role of nonlinear tempering in the stationary case. We study the interaction between attractive forces of anomalous aggregation and chemotaxis, with repulsive forces induced by nonlinear reactions. We show that this nonlinear interaction can prevent the phenomenon of anomalous aggregation when the local particle concentration grows too high. We also show that the effect of nonlinear tempering is to suppress the intermediate subdiffusive behaviour which results in a nonlinear advection diffusion equation in the long time limit.
\end{abstract}

Keywords and phrases: anomalous random walks, cell migration

Mathematics Subject Classification: 60G22, 82C31, 92B05

\section{Introduction}

Anomalous subdiffusive behaviour is characterised by a sublinear growth of the mean squared displacement in time $\left\langle x^{2}(t)\right\rangle \sim t^{\nu}, 0<\nu<1$. The parameter $\nu$ is known as the anomalous exponent. Subdiffusion in an observed natural phenomenon seen in areas as varied as transport of lipids on cell membranes [21], transport on fractal geometries [10], financial futures prices [18], signalling molecules in spiny dendrites $[9,20]$, and dispersive transport on amorphous semiconductors [22]. The standard model for subdiffusive transport is the continuous time random walk (CTRW) $[1,16,17]$.

CTRWs which include waiting time distributions with infinite mean lead to fractional equations in the diffusive limit, including the fractional Fokker-Planck equation (FFPE),

$$
\frac{\partial \rho(x, t)}{\partial t}=-\frac{\partial\left(v_{\nu}(x) \mathcal{D}_{t}^{1-\nu(x)} \rho\right)}{\partial x}+\frac{\partial^{2}\left(D_{\nu}(x) \mathcal{D}_{t}^{1-\nu(x)} \rho\right)}{\partial x^{2}}
$$

${ }^{*}$ Corresponding author. E-mail: steven.falconer@manchester.ac.uk 
see equation (38) in [4]. Equation (1.1) involves fractional diffusion coefficient $D_{\nu}(x)$ and fractional advection coefficient $v_{\nu}(x)$, as well as the Riemann-Liouville fractional derivative order $1-\nu(x)$,

$$
\mathcal{D}_{t}^{1-\nu(x)} \rho(x, t)=\frac{1}{\Gamma(\nu(x))} \frac{\partial}{\partial t} \int_{0}^{t} \frac{\rho(x, \tau) d \tau}{(t-\tau)^{1-\nu(x)}} .
$$

It has been shown that the fractional equations are not structurally stable with respect to spatial fluctuations in the anomalous exponent [4]. Fluctuations lead to the breakdown of the stationary behaviour, and the phenomenon of anomalous aggregation [2], where particles accumulate in a small region of space where the anomalous exponent is small compared to the surrounding region. To counteract this, models have been proposed which temper the anomalous waiting time of the CTRW in a physically justifiable way $[5,6]$. One such way is through nonlinear reactions which redistribute particles when the local concentration grows too high [15]. This normalises the subdiffusion, and allows for stationary behaviour to be realised once more.

The main aim of this paper is to implement nonlinear particle interactions into subdiffusive transport involving chemotaxis and nonlinear effects such as volume filling and adhesion. Chemotaxis describes a bias in the random movement of biological cells due to the concentration of an external signalling molecule. Volume filling and adhesion describe a bias in the movement either towards areas of either lower or higher cell concentrations. Several attempts have been made to take into account these effects in $[3,23]$ We introduce the additional nonlinear escape rate of particles which acts as a tempering to the anomalous trapping, characteristic of subdiffusion, and redistributes particles depending on the local mean field density. In this paper we use a rate based nonlinear random walk model involving residence time dependent escape rates and structural density of particles $[15,24,25]$. We apply standard model reduction procedure: starting from microscopic Markov model we managed to derive mesoscopic nonMarkovian master equations and then considered macroscopic (diffusion) limit. For the first time we derive generalised master equations, and diffusion approximations thereof, from the nonlinear random walk model involving separate modified nonlinear escape rates to the right and left. We introduce an additional instantaneous escape rate of particles dependent on the local mean field density. Here though the local mean field density is able to influence particles at any time, rather than just at the end of trapping events. Our flexible model allows for ease of extension to CTRW models which include more general transport operations than nearest neighbour jumps.

\section{Nonlinear interaction of non-Markovian random walkers}

In this paper we use a rate based nonlinear random walk model. A particle performs a random walk on a 1-D lattice, making instantaneous jumps from its location $x$ of length $l$ after random waiting times $T_{x}$. Here we consider the waiting times preceding jumps to the right and left as being separate and independent random variables, denoting the waiting time preceding a jump to the right $T_{x}^{\lambda}$ and a jump to the left $T_{x}^{\mu}$. If $T_{x}^{\mu}<T_{x}^{\lambda}$ the particle jumps to the left $x \rightarrow(x-l)$, and if $T_{x}^{\lambda}<T_{x}^{\mu}$ the particle jumps to the right $x \rightarrow(x+l)$. The actual time a particle rests for is then

$$
T_{x}=\min \left\{T_{x}^{\mu}, T_{x}^{\lambda}\right\}
$$

$T_{x}^{\lambda}$ and $T_{x}^{\mu}$ are drawn from the random waiting time PDFs $\psi_{\lambda}(x, \tau)$ and $\psi_{\mu}(x, \tau)$. The parameter $\tau$ is the residence time parameter, representing the length of time a particle has remained at the location $x$.

One can generalise this to allow transitions to other sites. Denote the waiting time of a transition from $x \rightarrow(x+i l)$ as $T_{x, i}^{\lambda}$ and from $x \rightarrow(x-i l)$ as $T_{x, i}^{\mu}$. Then the actual transition time happens at $T_{x}$ and is the minimum amongst the possible transitions to the left and right $T_{x}=\min \left\{\ldots, T_{x, n}^{\mu}, \ldots, T_{x, n}^{\lambda}, \ldots\right\}$. Some details are presented in Section $4 \mathrm{In}$ general, this random walk process is non-Markovian and known as a semi-Markov process, or generalised renewal process [14]. If $T_{x}$ is drawn from the exponential distribution, only then will the process be Markovian. 
The transition from one state to another can also be represented in the form of rates of escape, defined as instantaneous transition probabilities. The key fact of these escape rates is that they are dependent on the residence time variable $\tau$, making the process non-Markovian. The rate of transition from $x \rightarrow(x+l)$ is denoted by $\lambda(x, \tau)$, and from $x \rightarrow(x-l)$ is denoted by $\mu(x, \tau)$ :

$$
\lambda(x, \tau)=\lim _{\Delta t \rightarrow 0} \frac{\operatorname{Pr}\left\{T_{x}^{\lambda}<\tau+\Delta t \mid T_{x}^{\lambda}>\tau\right\}}{\Delta t}, \quad \mu(x, \tau)=\lim _{\Delta t \rightarrow 0} \frac{\operatorname{Pr}\left\{T_{x}^{\mu}<\tau+\Delta t \mid T_{x}^{\mu}>\tau\right\}}{\Delta t} .
$$

In survival analysis theory, this is known as the hazard rate function [19]. This way of thinking differs from standard random walk formulations involving the waiting time distribution. For escape rates which are constant with respect to residence time $\tau$, you have the classical Markov process which in the diffusive limit is approximated by the standard advection diffusion equation. If the escape rate is inversely proportional to the residence time, [4]: $\mu, \lambda \sim \nu / \tau, 0<\nu<1$, then the diffusive limit of such a process is the subdiffusive FFPE (1.1). Escape rates of this form mean walkers have a lower probability of escape for increasing residence time. This implies walkers experience long trapping events, with mean length $\bar{T}_{x}=\infty$. During these long trapping events it is an, implicit, assumption in other non-Markovian random walk models that walkers are protected from the influence of any external factors which would otherwise affect their transport. In the following subsection we describe a particle random walk model where the mean field density $\rho$ may influence the rate of escape of particles.

\subsection{Nonlinear interaction}

In this Subsection we will modify the escape rates of particles to the right and left. This is quite a flexible generalisation which allows for several macroscopic nonlinear effects to be described. We introduce another stochastic process acting independently of the non-Markovian trapping. This process allows the mean field density to influence the transport of particles even during long trapping events. This acts to counter the anomalous aggregation of particles present in subdiffusive random walk models $[2,4]$. We modify the escape rates $\lambda(x, \tau)$ and $\mu(x, \tau)$ as follows:

$$
\lambda_{\alpha}=\lambda(x, \tau)+\alpha_{\lambda}(\rho), \quad \mu_{\alpha}=\mu(x, \tau)+\alpha_{\mu}(\rho),
$$

where $\alpha_{\lambda}(\rho)$ and $\alpha_{\mu}(\rho)$ are the additional nonlinear escape rates to the right and left, with unspecified dependence upon $x$ and $t$. The probability of escape due to the nonlinear term is independent from the anomalous trapping, and in a time interval $\Delta t$ the probability is $\alpha(\rho) \Delta t$, where

$$
\alpha(\rho)=\alpha_{\lambda}(\rho)+\alpha_{\mu}(\rho) .
$$

The exact dependence of these nonlinear escape rates upon the mean field density leads to different qualitative macroscopic effects. Volume filling effects can be introduced with the following choices for $\lambda_{\alpha}$ and $\mu_{\alpha}$ :

$$
\lambda_{\alpha}=\lambda(x, \tau)+\alpha_{\lambda}(\rho(x+l, t)), \quad \mu_{\alpha}=\mu(x, \tau)+\alpha_{\mu}(\rho(x-l, t)) .
$$

Volume filling effects describe the model where diffusing particles have a non-zero volume, and that by occupying an area they may prevent other particles from doing the same. Volume filling describes the effect where diffusing particles have lower probability of jumping to the location where the density is higher [11,12]. This is modelled by imposing a dependence of the escape rate on the mean field density at the location the particle would escape to. The escape rate of a particle from $x \rightarrow(x+l)$ is dependent on the density of particles at $(x+l)$, with an increasing density lowering the escape rate. For example $\alpha(\rho)=1-\frac{\rho}{N}$, where $N$ represents the maximum density.

Adhesion effects can be introduced with the following choices for $\lambda_{\alpha}$ and $\mu_{\alpha}$ :

$$
\lambda_{\alpha}=\lambda(x, \tau)+\alpha_{\lambda}(\rho(x-l, t)), \quad \mu_{\alpha}=\mu(x, \tau)+\alpha_{\mu}(\rho(x+l, t)) .
$$


Adhesion effects describe the sticking together of particles [13]. The escape rate of a particle from $x \rightarrow(x+l)$ is dependent on the density of particles at $(x-l)$, with an increasing density lowering the escape rate.

A dependence upon the local gradient of density [12] could be modelled also:

$$
\lambda_{\alpha}=\lambda(x, \tau)+\kappa\left(\alpha_{\lambda}(\rho(x+l, t))-\alpha_{\lambda}(\rho(x, t))\right), \quad \mu_{\alpha}=\mu(x, \tau)+\kappa\left(\alpha_{\mu}(\rho(x, t))-\alpha_{\mu}(\rho(x-l, t))\right) .
$$

In the subdiffusive case, where $\lambda, \mu \sim 1 / \tau$ the nonlinear terms $\alpha_{\mu}$ and $\alpha_{\lambda}$ have the effect of tempering factors preventing particles from being trapped for infinite times.

\subsection{Structured density of particles}

In order to derive a master equation for the evolution of the non-Markovian process let us introduce the structured number density of particles $[15,24,25] \xi(x, t, \tau)$ at location $x$, at time $t$, with residence time $\tau$. The described random walk model above leads to the balance equation for $\xi$,

$$
\frac{\partial \xi}{\partial t}+\frac{\partial \xi}{\partial \tau}=-\left(\lambda(x, \tau)+\alpha_{\lambda}(\rho)\right) \xi(x, t, \tau)-\left(\mu(x, \tau)+\alpha_{\mu}(\rho)\right) \xi(x, t, \tau)
$$

and the objective is to derive a master equation for the unstructured density $\rho(x, t)$

$$
\rho(x, t)=\int_{0}^{t} \xi(x, t, \tau) d \tau .
$$

This approach has been used by many authors $[2,4,7,15,24,25]$ for the study of non-Markovian random walks, and has recently been found to be one of the most suitable for further nonlinear generalisations [3, $6,8,23]$.

Firstly, we define some key quantities from the random walk model. The survival functions PDFs for jumps to the right and left are denoted,

$$
\Psi_{\lambda}(x, \tau)=\operatorname{Pr}\left\{T_{x}^{\lambda}>\tau\right\}, \quad \Psi_{\mu}(x, \tau)=\operatorname{Pr}\left\{T_{x}^{\mu}>\tau\right\},
$$

and define the probability a particle remains at $x$ for a time $\tau$. These are related to the waiting time PDFs through,

$$
\Psi_{\lambda}(x, \tau)=\int_{\tau}^{\infty} \psi_{\lambda}(x, s) d s, \quad \Psi_{\mu}(x, \tau)=\int_{\tau}^{\infty} \psi_{\mu}(x, s) d s
$$

where,

$$
\psi_{\lambda}(x, \tau)=\lim _{\Delta t \rightarrow 0} \frac{\operatorname{Pr}\left\{\tau<T_{x}^{\lambda}<\tau+\Delta t\right\}}{\Delta t}, \quad \psi_{\mu}(x, \tau)=\lim _{\Delta t \rightarrow 0} \frac{\operatorname{Pr}\left\{\tau<T_{x}^{\mu}<\tau+\Delta t\right\}}{\Delta t},
$$

are the PDFs for the waiting times for jumps to the right and left, respectively. Bayes' Theorem allows us to relate the escape rates to both the waiting time PDFs,

$$
\lambda(x, \tau)=\frac{\psi_{\lambda}(x, \tau)}{\Psi(x, \tau)}, \quad \mu(x, \tau)=\frac{\psi_{\mu}(x, \tau)}{\Psi(x, \tau)},
$$

where from the definition of $T_{x}=\min \left\{T_{x}^{\lambda}, T_{x}^{\mu}\right\}$, we can write the total survival PDF as

$$
\begin{aligned}
\Psi(x, \tau) & =\operatorname{Pr}\left\{\min \left\{T_{x}^{\lambda}, T_{x}^{\mu}\right\}>\tau\right\} \\
& =\Psi_{\lambda}(x, \tau) \Psi_{\mu}(x, \tau) \\
& =e^{-\int_{0}^{\tau}(\lambda(x, s)+\mu(x, s)) d s},
\end{aligned}
$$

with the final equality following from (2.3) and (2.5). Differentiation of this equation with respect to $\tau$ gives the total waiting time PDF,

$$
\psi(x, \tau)=\psi_{\lambda}(x, \tau)+\psi_{\mu}(x, \tau)
$$


where waiting time densities $\psi_{\lambda}(x, \tau)$ and $\psi_{\mu}(x, \tau)$ are related by,

$$
\psi_{\lambda}(x, \tau)=-\frac{\partial \Psi_{\mu}(x, \tau)}{\partial \tau} \Psi_{\lambda}(x, \tau), \quad \psi_{\mu}(x, \tau)=-\frac{\partial \Psi_{\lambda}(x, \tau)}{\partial \tau} \Psi_{\mu}(x, \tau)
$$

In the next Section we will derive the non-Markovian master equation for the random walk model.

\section{Nonlinear master equation}

The balance equation (2.1) is written in terms of a sum of the two types of escape rates, the anomalous escape rates $\lambda(x, \tau)+\mu(x, \tau)$ and the additional escape rate $\alpha_{\lambda}(\rho)+\alpha_{\mu}(\rho)$. The initial condition at $t=0$ is given by:

$$
\xi(x, 0, \tau)=\rho_{0}(x) \delta(\tau),
$$

where $\rho_{0}(x)$ is the initial density. The boundary condition for $\xi(x, t, \tau)$ at zero residence time $\tau=0$ is:

$$
\xi(x, t, 0)=\int_{0}^{t}\left[\lambda(x-l, \tau)+\alpha_{\lambda}(\rho)\right] \xi(x-l, t, \tau) d \tau+\int_{0}^{t}\left[\mu(x+l, \tau)+\alpha_{\mu}(\rho)\right] \xi(x+l, t, \tau) d \tau .
$$

The number density of particles with residence time $\tau$ escaping from $x \rightarrow(x+l)$ per unit time is $\left[\lambda(x, \tau)+\alpha_{\lambda}(\rho)\right] \xi(x, t, \tau)$, from $x \rightarrow(x-l)$ is $\left[\mu(x, \tau)+\alpha_{\mu}(\rho)\right] \xi(x, t, \tau)$. The total escape rate to the right and left, respectively, is found by integrating over all $\tau$,

$$
\begin{aligned}
& i_{\lambda}(x, t)=\int_{0}^{t}\left[\lambda(x, \tau) \xi(x, t, \tau) d \tau+\alpha_{\lambda}(\rho)\right] \rho(x, t), \\
& i_{\mu}(x, t)=\int_{0}^{t}\left[\mu(x, \tau) \xi(x, t, \tau) d \tau+\alpha_{\mu}(\rho)\right] \rho(x, t),
\end{aligned}
$$

with the second terms in both equations due to equation (2.2). Solving equation (2.1) by method of characteristics yields, for $\tau<t$,

$$
\xi(x, t, \tau)=\xi(x, t-\tau, 0) e^{-\int_{0}^{\tau}(\lambda(x, s)+\mu(x, s)) d s} \frac{e^{\Phi(x, t-\tau)}}{e^{\Phi(x, t)}},
$$

where,

$$
\Phi(t)=\int_{0}^{t}\left[\alpha_{\lambda}(\rho)+\alpha_{\mu}(\rho)\right] d s .
$$

Let us denote the integral arrival rate of particles arriving at $x$ exactly at $t$ as $j(x, t)$, equal to boundary condition (3.1),

$$
j(x, t)=\xi(x, t, 0),
$$

which, with equations (3.2), can be written as,

$$
j(x, t)=i_{\lambda}(x-l, t)+i_{\mu}(x+l, t) .
$$

With characteristic solution (3.4) this becomes,

$$
\begin{aligned}
j(x, t)= & e^{-\Phi(t)} \int_{0}^{t} \psi_{\lambda}(x-l, \tau) j(x-l, t-\tau) e^{\Phi(t-\tau)} d \tau+\rho_{0}(x-l) \psi_{\lambda}(x-l, t) e^{-\Phi(t)} \\
& +\alpha_{\lambda}(\rho) \rho(x-l, t) \\
+ & e^{-\Phi(t)} \int_{0}^{t} \psi_{\mu}(x+l, \tau) j(x+l, t-\tau) e^{\Phi(t-\tau)} d \tau+\rho_{0}(x+l) \psi_{\mu}(x+l, t) e^{-\Phi(t)} \\
& +\alpha_{\mu}(\rho) \rho(x+l, t),
\end{aligned}
$$


with second terms $\rho_{0}(x)$ coming from the contribution of $\left.\xi(x, t, \tau)\right|_{\tau=t}$ due to the singularity of the initial condition. Inserting the expression (3.4) for $\xi(x, t, \tau)$ into the expression for the unstructured density $(2.2)$ $\rho$ :

$$
\rho(x, t)=e^{-\Phi(t)} \int_{0}^{t} \Psi(x, \tau) j(x, t-\tau) e^{\Phi(t-\tau)} d \tau+\rho_{0}(x) \Psi(x, t) e^{-\Phi(t)} .
$$

Using equation (3.4) one can write the integral escape rates to the right and left respectively as

$$
\begin{aligned}
& i_{\lambda}(x, t)=e^{-\Phi(t)} \int_{0}^{t} \psi_{\lambda}(x, \tau) j(x, t-\tau) e^{\Phi(t-\tau)} d \tau+\rho_{0}(x) \psi_{\lambda}(x, t) e^{-\Phi(t)}+\alpha_{\lambda}(\rho) \rho(x, t), \\
& i_{\mu}(x, t)=e^{-\Phi(t)} \int_{0}^{t} \psi_{\mu}(x, \tau) j(x, t-\tau) e^{\Phi(t-\tau)} d \tau+\rho_{0}(x) \psi_{\mu}(x, t) e^{\Phi(t)}+\alpha_{\mu}(\rho) \rho(x, t) .
\end{aligned}
$$

To eliminate $j(x, t)$, Laplace transforms of these equations (3.8) together with equation (3.7) yield expressions for integral escape rates:

$$
\begin{aligned}
& i_{\lambda}(x, t)=e^{-\Phi(t)} \int_{0}^{t} K_{\lambda}(x, t-\tau) \rho(x, \tau) e^{\Phi(\tau)} d \tau+\alpha_{\lambda}(\rho(x, t)) \rho(x, t), \\
& i_{\mu}(x, t)=e^{-\Phi(t)} \int_{0}^{t} K_{\mu}(x, t-\tau) \rho(x, \tau) e^{\Phi(\tau)} d \tau+\alpha_{\mu}(\rho(x, t)) \rho(x, t) .
\end{aligned}
$$

Here $K_{\mu}(x, t)$ and $K_{\lambda}(x, t)$ are the space dependent memory kernels, defined in terms of their Laplace transforms as,

$$
\hat{K}_{\lambda}(x, s)=\frac{\hat{\psi}_{\lambda}(x, s)}{\hat{\Psi}(x, s)}, \quad \hat{K}_{\mu}(x, s)=\frac{\hat{\psi}_{\mu}(x, s)}{\hat{\Psi}(x, s)} .
$$

By differentiating equation (2.2) and using the balance equation for $\xi(x, t, \tau)(2.1)$, we find that the master equation for the unstructured density can be written as a balance of escape and arrival rates:

$$
\frac{\partial \rho}{\partial t}=j(x, t)-i_{\lambda}(x, t)-i_{\mu}(x, t)
$$

Due to conservation, the arrival rate of particles to $x$ is the sum of escapes from $(x \pm l)$, equation (3.6) for $j(x, t)$

$$
\frac{\partial \rho}{\partial t}=i_{\lambda}(x-l, t)+i_{\mu}(x+l, t)-i_{\lambda}(x, t)-i_{\mu}(x, t) .
$$

This is simply the balance of particles arriving at and leaving the point $x$. So that by inserting expressions (3.9) for $i_{\lambda}$ and $i_{\mu}$ we can write the full form of the generalised master equation for our random walk scheme,

$$
\begin{aligned}
\frac{\partial \rho(x, t)}{\partial t}= & e^{-\Phi(t)} \int_{0}^{t} K_{\lambda}(x-l, t-\tau) \rho(x-l, \tau) e^{\Phi(\tau)} d \tau+\alpha_{\lambda}(\rho(x-l, t)) \rho(x-l, t) \\
& +e^{-\Phi(t)} \int_{0}^{t} K_{\mu}(x+l, t-\tau) \rho(x+l, \tau) e^{\Phi(\tau)} d \tau+\alpha_{\mu}(\rho(x+l, t)) \rho(x+l, t) \\
& -e^{-\Phi(t)} \int_{0}^{t} K(x, t-\tau) \rho(x, \tau) e^{\Phi(\tau)} d \tau-\alpha(\rho(x, t)) \rho(x, t)
\end{aligned}
$$

\section{Multiple escape rates}

In this section we show how we can generalise the model easily to allow transitions to occur not only between neighbouring lattice sites. Assuming the same procedure, that is the particle jumps a length $i l$ 
to one of the $n$ sites to the left or $n$ sites to the right after a time $T_{x, i}^{\lambda}$ or $T_{x, i}^{\mu}$. The total waiting time in this case would be, analogously to (2.1),

$$
T_{x}=\min \left\{T_{x, n}^{\mu}, T_{x,(n-1)}^{\mu}, \ldots, T_{x,(n-1)}^{\lambda}, T_{x, n}^{\lambda}\right\} .
$$

The random waiting times $T_{x, i}^{\lambda}$ and $T_{x, i}^{\mu}$ are distributed as $\psi_{i}^{\lambda}(x, \tau)$ and $\psi_{i}^{\mu}(x, \tau)$ respectively. The transition rate, in addition, is defined to be $\mu_{i}(x, \tau)$ for jumping from $x \rightarrow(x-i l)$ and $\lambda_{i}(x, \tau)$ for jumping from $x \rightarrow(x+i l), i=1, \ldots, n$. These are again modified:

$$
\lambda_{\alpha, i}=\lambda_{i}(x, \tau)+\alpha_{\lambda, i}(\rho(x, t)), \quad \mu_{\alpha, i}=\mu_{i}(x, \tau)+\alpha_{\mu, i}(\rho(x, t)) .
$$

Then the balance equation for the structured density $\xi(x, t, \tau)$ now reads,

$$
\frac{\partial \xi}{\partial t}+\frac{\partial \xi}{\partial \tau}=-\sum_{i=1}^{n}\left[\lambda_{i}(x, \tau)+\alpha_{\lambda, i}(\rho(x, t))\right] \xi(x, t, \tau)-\sum_{i=1}^{n}\left[\mu_{i}(x, \tau)+\alpha_{\mu, i}(\rho(x, t))\right] \xi(x, t, \tau) .
$$

All other relevant quantities can be defined in an analogous way as previously.

There are now $2 n$ memory kernels, for $1 \leq i \leq n$ :

$$
\hat{K}_{i}^{\lambda}(x, s)=\frac{\hat{\psi}_{i}^{\lambda}(x, s)}{\hat{\Psi}(x, s)}, \quad \hat{K}_{i}^{\mu}(x, s)=\frac{\hat{\psi}_{i}^{\mu}(x, s)}{\hat{\Psi}(x, s)},
$$

where the total survival PDF $\Psi(x, \tau)$ defined by:

$$
\begin{aligned}
\Psi(x, \tau) & =\prod_{i=1}^{n} \Psi_{i}^{\lambda}(x, \tau) \times \prod_{j=1}^{n} \Psi_{j}^{\mu}(x, \tau) \\
& =e^{-\int_{0}^{t}\left[\sum_{i=1}^{n} \lambda_{i}(x, s)+\sum_{j=1}^{n} \mu_{j}(x, s)\right] d s} .
\end{aligned}
$$

The integral escape rates to the right and left $i_{\lambda}(x, t)$ and $i_{\mu}(x, t)$ are sums of the escape rates to each site to the right and left of $x$ :

$$
\begin{aligned}
& i_{\lambda}(x, t)=\sum_{i=1}^{n}\left(e^{-\Phi(x, t)} \int_{0}^{t} K_{i}^{\lambda}(x, t-\tau) \rho(x, \tau) e^{\Phi(x, \tau)} d \tau+\alpha_{\lambda, i}(\rho(x, t)) \rho(x, t)\right), \\
& i_{\mu}(x, t)=\sum_{i=1}^{n}\left(e^{-\Phi(x, t)} \int_{0}^{t} K_{i}^{\mu}(x, t-\tau) \rho(x, \tau) e^{\Phi(x, \tau)} d \tau+\alpha_{\mu, i}(\rho(x, t)) \rho(x, t)\right) .
\end{aligned}
$$

So the master equation is written in terms of a sum over the escape related quantities of the memory kernel and additional escape tempering term:

$$
\begin{aligned}
\frac{\partial \rho}{\partial t}=\sum_{i=1}^{n}\left(e^{-\Phi(x-i l, t)} \int_{0}^{t} K_{i}^{\lambda}(x-i l, t-\tau) \rho(x-i l, \tau) e^{\Phi(x-i l, t-\tau)} d \tau\right. \\
\left.\quad+\alpha_{\lambda, i}(\rho(x-i l, t)) \rho(x-i l, t)\right) \\
+\sum_{i=1}^{n}\left(e^{-\Phi(x+i l, t)} \int_{0}^{t} K_{i}^{\mu}(x+i l, t-\tau) \rho(x+i l, \tau) e^{\Phi(x+i l, t-\tau)} d \tau\right. \\
\left.\quad+\alpha_{\mu, i}(\rho(x+i l, t)) \rho(x+i l, t)\right) \\
-e^{-\Phi(x, t)} \int_{0}^{t} K(x, t-\tau) \rho(x, \tau) e^{\Phi(x, t-\tau)} d \tau+\alpha(\rho(x, t)) \rho(x, t)
\end{aligned}
$$

where $\alpha(\rho(x, t))=\sum_{i=1}^{n}\left(\alpha_{\lambda, i}(\rho(x, t))+\alpha_{\mu, i}(\rho(x, t))\right)$. 


\section{Tempered Anomalous Subdiffusion}

In this section we derive the fractional master equation as a special case of equation (3.11). When escape rates are inversely proportional to residence time $\tau$ the memory kernel becomes a time fractional operator. We assume that the jumping rates are defined as:

$$
\lambda(x, \tau)=\frac{\nu_{\lambda}(x)}{\tau_{0}(x)+\tau}, \quad \mu(x, \tau)=\frac{\nu_{\mu}(x)}{\tau_{0}(x)+\tau} .
$$

Using the definition of the survival function (2.5), we find the survival functions have a power-law dependence,

$$
\Psi_{\lambda}(x, \tau)=\left[\frac{\tau_{0}(x)}{\tau_{0}(x)+\tau}\right]^{\nu_{\lambda}(x)}, \Psi_{\mu}(x, \tau)=\left[\frac{\tau_{0}(x)}{\tau_{0}(x)+\tau}\right]^{\nu_{\mu}(x)},
$$

and thus the total survival probability can be written as:

$$
\Psi(x, \tau)=\left(\frac{\tau_{0}(x)}{\tau_{0}(x)+\tau}\right)^{\nu(x)},
$$

where $\nu(x)=\nu_{\lambda}(x)+\nu_{\mu}(x)$ depends on the spatial variable $x$. The total waiting time pdf defined in equation (2.6) has the Pareto form:

$$
\psi(x, \tau)=\frac{\nu(x) \tau_{0}(x)^{\nu(x)}}{\left(\tau_{0}(x)+\tau\right)^{1+\nu(x)}} .
$$

Let us introduce the probabilities of jumping to the right and left, as the ratio of $\lambda(x, \tau)$ and $\mu(x, \tau)$ to $\lambda(x, \tau)+\mu(x, \tau)$, that are independent of the residence time $\tau$, as:

$$
p_{\lambda}(x)=\frac{\nu_{\lambda}(x)}{\nu(x)}, \quad p_{\mu}(x)=\frac{\nu_{\mu}(x)}{\nu(x)} .
$$

The transition PDFs $\psi_{\lambda}(x, \tau)=\lambda(x, \tau) \Psi(x, \tau)$ and $\psi_{\mu}(x, \tau)=\mu(x, \tau) \Psi(x, \tau)$ can be rewritten in terms of the jumping probability as:

$$
\psi_{\lambda}(x, \tau)=p_{\lambda}(x) \psi(x, \tau), \quad \psi_{\mu}(x, \tau)=p_{\mu}(x) \psi(x, \tau) .
$$

In the limit of $t \rightarrow \infty$, by the Tauberian theorem, the memory kernels (3.10) have asymptotic approximations in Laplace space as $s \rightarrow 0$ :

$$
\hat{K}_{\lambda}(x, s) \simeq \frac{p_{\lambda}(x) s^{1-\nu(x)}}{g(x)}, \quad \hat{K}_{\mu}(x, s) \simeq \frac{p_{\mu}(x) s^{1-\nu(x)}}{g(x)},
$$

where,

$$
g(x)=\Gamma(1-\nu(x)) \tau_{0}(x)^{\nu(x)} .
$$

A combined memory kernel can be defined from (5.1) and (5.2),

$$
\begin{aligned}
\hat{K}(x, s) & =\hat{K}_{\lambda}(x, s)+\hat{K}_{\mu}(x, s) \\
& =\frac{s^{1-\nu(x)}\left[p_{\lambda}(x)+p_{\mu}(x)\right]}{g(x)}=\frac{s^{1-\nu(x)}}{g(x)} .
\end{aligned}
$$

Using the expressions for the memory kernels (5.3), the integral escape rates to the right and left are:

$$
\begin{aligned}
& i_{\lambda}(x, t)=a(x) e^{-\Phi(x, t)} \mathcal{D}_{t}^{1-\nu(x)}\left[\rho(x, t) e^{\Phi(x, t)}\right]+\alpha_{\lambda}(\rho(x, t)) \rho(x, t), \\
& i_{\mu}(x, t)=b(x) e^{-\Phi(x, t)} \mathcal{D}_{t}^{1-\nu(x)}\left[\rho(x, t) e^{\Phi(x, t)}\right]+\alpha_{\mu}(\rho(x, t)) \rho(x, t),
\end{aligned}
$$


where $\mathcal{D}_{t}^{1-\nu(x)}$ is the space dependent Riemann-Liouville fractional derivative (1.2) of order $(1-\nu(x))$ and $a(x)$ and $b(x)$ are the anomalous rate coefficients,

$$
a(x)=\frac{p_{\lambda}(x)}{g(x)}=\frac{\nu_{\lambda}(x)}{\nu(x) \Gamma(1-\nu(x)) \tau_{0}(x)^{\nu(x)}}, \quad b(x)=\frac{p_{\mu}(x)}{g(x)}=\frac{\nu_{\mu}(x)}{\nu(x) \Gamma(1-\nu(x)) \tau_{0}(x)^{\nu(x)}} .
$$

The fractional master equation then can be found:

$$
\begin{aligned}
\frac{\partial \rho(x, t)}{\partial t}= & a(x-l) e^{-\Phi(x-l, t)} \mathcal{D}_{t}^{1-\nu(x-l)}\left[\rho(x-l, t) e^{\Phi(x-l, t)}\right]+\alpha_{\lambda}(\rho(x-l, t)) \rho(x-l, t) \\
& +b(x+l) e^{-\Phi(x+l, t)} \mathcal{D}_{t}^{1-\nu(x+l)}\left[\rho(x+l, t) e^{\Phi(x+l, t)}\right]+\alpha_{\mu}(\rho(x+l, t)) \rho(x+l, t) \\
& -(a(x)+b(x)) e^{-\Phi(x, t)} \mathcal{D}_{t}^{1-\nu(x)}\left[\rho(x, t) e^{\Phi(x, t)}\right]-\alpha(\rho(x, t)) \rho(x, t) .
\end{aligned}
$$

\section{Diffusion limit}

In the limit as $l \rightarrow 0$ we can find, by Taylor series expansion, the nonlinear fractional Fokker-Planck equation

$$
\frac{\partial \rho(x, t)}{\partial t}=-l \frac{\partial}{\partial x}\left[i_{\lambda}(x, t)-i_{\mu}(x, t)\right]+\frac{l^{2}}{2} \frac{\partial^{2}}{\partial x^{2}}\left[i_{\lambda}(x, t)+i_{\mu}(x, t)\right],
$$

for $i_{\lambda}(x, t)$ and $i_{\mu}(x, t)$ defined in (5.4).

In what follows, let us consider the model for which the jump probabilities $p_{\lambda}(x)$ and $p_{\mu}(x)$ depend on the chemotactic substance $S(x)$ as follows:

$$
p_{\lambda}(x)=A e^{-\beta(S(x+l)-S(x))}, \quad p_{\mu}(x)=A e^{-\beta(S(x-l)-S(x))},
$$

where the parameter $A$ satisfies the probability conservation $p_{\lambda}(x)+p_{\mu}(x)=1$. These jump probabilities describe the bias of cells' movements with respect to the difference of local concentration of chemotactic substance. The difference satisfies:

$$
p_{\lambda}(x)-p_{\mu}(x)=\frac{e^{-\beta S(x+l)}-e^{-\beta S(x-l)}}{e^{-\beta S(x+l)}+e^{-\beta S(x-l)}},
$$

and in the limit $l \rightarrow 0$ we have the standard chemotaxis model,

$$
a(x)-b(x)=\frac{p_{\lambda}(x)-p_{\mu}(x)}{g(x)}=-\frac{l \beta}{g(x)} \frac{d S(x)}{d x}+\mathrm{o}(l) .
$$

We now choose particular escape rates simply based on the local mean field density. The effect is particles will be more likely to escape from an area of high concentration, and this will counteract any crowding effects:

$$
\lambda_{\alpha}(x, \tau)=\lambda(x, \tau)+\alpha_{\lambda}(\rho(x, t)), \quad \mu_{\alpha}(x, \tau)=\mu(x, \tau)+\alpha_{\mu}(\rho(x, t)) .
$$

We can organise the difference between $\alpha_{\lambda}$ and $\alpha_{\mu}$ in an analogous manner. If we write,

$$
p_{\alpha, \lambda}(x, t)=\frac{\alpha_{\lambda}(\rho(x, t))}{\alpha(\rho(x, t))}, \quad p_{\alpha_{\mu}}(x, t)=\frac{\alpha_{\mu}(\rho(x, t))}{\alpha(\rho(x, t))},
$$

and choose $\alpha_{\lambda}$ and $\alpha_{\mu}$ such that,

$$
p_{\alpha, \lambda}(x, t)=B e^{-\kappa(\rho(x+l, t)-\rho(x, t))}, \quad p_{\alpha_{\mu}}(x, t)=B e^{-\kappa(\rho(x-l, t)-\rho(x, t))},
$$

where $B$ satisfies $p_{\alpha, \lambda}(x, t)+p_{\alpha_{\mu}}(x, t)=1$. We can approximate the difference:

$$
p_{\alpha, \lambda}(x, t)-p_{\alpha_{\mu}}(x, t)=-l \kappa \frac{\partial \rho}{\partial x}+\mathrm{o}(l) .
$$


We then obtain the nonlinear fractional equation written in full:

$$
\begin{aligned}
\frac{\partial \rho(x, t)}{\partial t}= & \frac{\partial}{\partial x}\left[\frac{l^{2} \beta}{g(x)} \frac{d S}{d x} e^{-\Phi(x, t)} \mathcal{D}_{t}^{1-\nu(x)} \rho(x, t) e^{\Phi(x, t)}-l^{2} \kappa \frac{\partial \rho}{\partial x} \alpha(\rho(x, t))\right] \\
& +\frac{\partial^{2}}{\partial x^{2}}\left[\frac{l^{2}}{2 g(x)} e^{-\Phi(x, t)} \mathcal{D}_{t}^{1-\nu(x)} \rho(x, t) e^{\Phi(x, t)}+\alpha(\rho(x, t)) \rho(x, t)\right] .
\end{aligned}
$$

Notice here that it is not possible to separate the additional escape from the subdiffusive transport terms, with the former appearing as a convolution under the fractional derivative operator. The transport and diffusion are controlled both by the chemotaxis and the local gradient of the mean field.

\subsection{Linear tempering}

In this subsection, we wish to find the stationary version of equation (6.1) when the additional nonlinear escape rates $\alpha_{\lambda}(\rho(x, t))$ and $\alpha_{\mu}(\rho(x, t))$ are independent of $\rho$. Now $\Phi(x, t)=\int_{0}^{t} \alpha(\rho(x, s)) d s=\alpha(x) t$ and the fractional escape rates (5.4) become:

$$
\begin{aligned}
& i_{\lambda}(x, t)=a(x) e^{-\alpha(x) t} \mathcal{D}_{t}^{1-\nu(x)}\left[\rho(x, t) e^{\alpha(x) t}\right]+\alpha_{\lambda}(x) \rho(x, t), \\
& i_{\mu}(x, t)=b(x) e^{-\alpha(x) t} \mathcal{D}_{t}^{1-\nu(x)}\left[\rho(x, t) e^{\alpha(x) t}\right]+\alpha_{\mu}(x) \rho(x, t) .
\end{aligned}
$$

The stationary density is defined in limit $s \rightarrow 0$ as,

$$
\rho_{\mathrm{st}}(x)=\lim _{s \rightarrow 0} s \hat{\rho}(x, s),
$$

and the stationary escape rates as,

$$
i_{\lambda, \mathrm{st}}(x)=\lim _{s \rightarrow 0} s \hat{i}_{\lambda}(x, s), \quad i_{\mu, \mathrm{st}}(x)=\lim _{s \rightarrow 0} s \hat{i}_{\mu}(x, s) .
$$

By the shift theorem, the Laplace transforms of equations (6.3) are:

$$
\begin{aligned}
& \hat{i}_{\lambda}(x, s)=a(x)[s+\alpha(x)]^{1-\nu(x)} \hat{\rho}(x, s)+\alpha_{\lambda}(x) \hat{\rho}(x, s), \\
& \hat{i}_{\mu}(x, s)=b(x)[s+\alpha(x)]^{1-\nu(x)} \hat{\rho}(x, s)+\alpha_{\mu}(x) \hat{\rho}(x, s) .
\end{aligned}
$$

Then the stationary escape rates can be written in the Markovian form,

$$
\begin{aligned}
& i_{\lambda, \mathrm{st}}(x)=\lambda_{\nu}(x) \rho_{\mathrm{st}}(x), \\
& i_{\mu, \mathrm{st}}(x)=\mu_{\nu}(x) \rho_{\mathrm{st}}(x),
\end{aligned}
$$

where

$$
\begin{aligned}
& \mu_{\nu}(x)=b(x) \alpha(x)^{1-\nu(x)}+\alpha_{\mu}(x), \\
& \lambda_{\nu}(x)=a(x) \alpha(x)^{1-\nu(x)}+\alpha_{\lambda}(x) .
\end{aligned}
$$

The stationary limit of nonlinear fractional equation (6.1) reduces to the $2^{\text {nd }}$ order ODE

$$
\frac{\partial}{\partial x}\left(v_{\nu}(x) \rho_{\mathrm{st}}(x)\right)=\frac{\partial^{2}}{\partial x^{2}}\left(D_{\nu}(x) \rho_{\mathrm{st}}(x)\right),
$$

where,

$$
D_{\nu}(x)=\frac{l^{2}}{2}\left(\frac{\left(\tau_{0}(x) \alpha(x)\right)^{1-\nu(x)}}{\Gamma(1-\nu(x)) \tau_{0}(x)}+\alpha(x)\right), \quad v_{\nu}(x)=D_{\nu}(x) 2 \beta \frac{d S(x)}{d x}
$$

with,

$$
\frac{d S(x)}{d x}=\frac{2}{l} \frac{\lambda_{\nu}(x)-\mu_{\nu}(x)}{\mu_{\nu}(x)+\lambda_{\nu}(x)}
$$




\subsection{Nonlinear tempering}

If a stationary distribution exists, then the tempering parameter $e^{-\int_{0}^{t} \alpha(\rho(x, s)) d s}$ can be written $e^{-\alpha\left(\rho_{\text {st }}(x)\right) t}$ as $t \rightarrow \infty$. Following the same procedure as for the linear case in the previous subsection, we arrive at the stationary nonlinear fractional equation:

$$
\frac{\partial}{\partial x}\left(v_{\nu}\left(\rho_{\mathrm{st}}, x\right) \rho_{\mathrm{st}}(x)\right)=\frac{\partial^{2}}{\partial x^{2}}\left(D_{\nu}\left(\rho_{\mathrm{st}}, x\right) \rho_{\mathrm{st}}(x)\right),
$$

where,

$$
v_{\nu}\left(\rho_{\mathrm{st}}, x\right)=\frac{l \beta}{g(x)} \frac{d S(x)}{d x} \alpha\left(\rho_{\mathrm{st}}\right)^{1-\nu(x)}+l \kappa \frac{d \rho_{\mathrm{st}}}{d x} \alpha\left(\rho_{\mathrm{st}}\right)
$$

and,

$$
D_{\nu}\left(\rho_{\mathrm{st}}, x\right)=\frac{l^{2}}{2}\left(\frac{\left(\tau_{0}(x) \alpha\left(\rho_{\mathrm{st}}\right)\right)^{1-\nu(x)}}{\Gamma(1-\nu(x)) \tau_{0}(x)}+\alpha\left(\rho_{\mathrm{st}}\right)\right) .
$$

Note that the velocity depends on the difference $\alpha_{\lambda}(x)-\alpha_{\mu}(x)=-l \kappa \frac{d \rho_{\mathrm{st}}}{d x} \alpha\left(\rho_{\mathrm{st}}(x)\right)$. So the shape of the mean field density can influence the transport.

This equation can be solved by direct Monte Carlo simulation of fractional master equation (3.11), or by simulation of Markovian evolution equation from which balance equation (2.1) is derived:

$$
\xi(x, t+\Delta t, \tau+\Delta \tau)=\xi(x, t, \tau)(1-\lambda(x, \tau) \Delta \tau-\mu(x, \tau) \Delta \tau)\left(1-\alpha_{\lambda}(\rho) \Delta t-\alpha_{\mu}(\rho) \Delta t\right) .
$$

The simulated result is shown in Fig. 1. In this particular case, chemotaxis forces drive particles from the right to the left. In the absence of tempering, case $A$, we have the decaying aggregation pattern with maximum at the boundary $x=0$. In the nonlinear cases $B$ and $C$ we observe the interesting phenomena of the particles being driven to the right and finding a new local maximum. The is purely a result of the nonlinear interaction.

\section{Conclusion}

In this paper we studied a non-Markovian random walk model which included nonlinear particle interactions and chemotactic forcing. The nonlinear particle interactions were introduced to the model through the modified escape rates (2.1). We derived the general non-Markovian master equation which included the exponential factors involving the nonlinear escape rates. In the subdiffusive case, the master equation includes the Riemann-Liouville fractional derivative with the nonlinear factors acting as a tempering to the anomalous trapping mechanism.

In the diffusive limit, $l \rightarrow 0$, we have a nonlinear fractional equation (6.2). In the long time stationary limit, the diffusion coefficient includes both the rate of additional escape $\alpha$ and subdiffusive anomalous exponent $\nu(x)$. We find that if the rate $\alpha$ is independent of the mean field density, the stationary solution can be found to be of Boltzmann type, and anomalous aggregation is not observed. In the case of nonlinear dependence of $\alpha$ upon $\rho$ we have also derived a stationary nonlinear equation (6.5). The main result of this paper is that the nonlinear tempering due to nonlinear escape, (2.2), leads to nonlinear advection and diffusion. One can see from (6.6) and (6.7) that advection and diffusion depend on nonlinear escape rate $\alpha(\rho)$. In Section 2.1 we discuss how $\alpha(\rho)$ relates to volume filling and adhesion.

This nonlinear fractional equations could be solved by direct Monte Carlo simulation of the master equation (5.5). We have simulated a particular case of tempered anomalous subdiffusion, in Fig 1.

Acknowledgements. Sergei Fedotov is grateful for support by the Engineering and Physical Sciences Research Council (EPSRC) through grant EP/J019526/1. We thank Nickolay Korabel and Peter Straka for helpful discussions. 


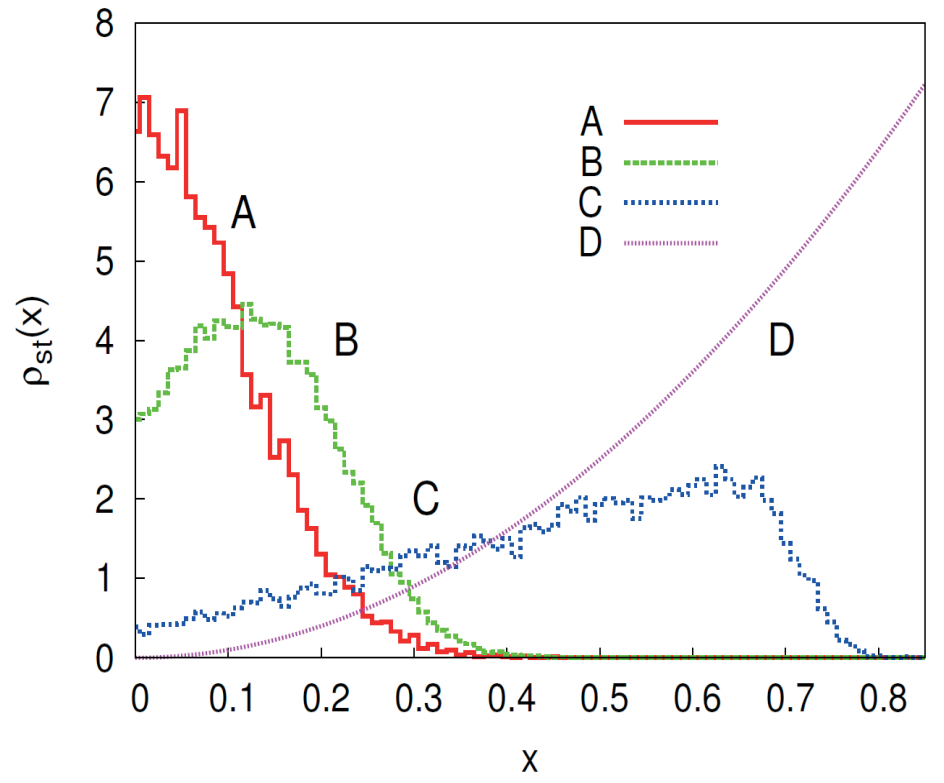

FiguRE 1. Simulated stationary profiles $\rho_{\text {st }}(x)$ for non-Markovian random walk eq. (6.8) in quadratic stationary chemotactic profile, $\mathrm{D}, S(x)=0.5 x^{2}$ on bounded domain $[0,1]$ with no-flux boundary conditions. Rates: $\lambda(x, \tau)=\left(\frac{1}{2}-l \frac{d S(x)}{d x}\right) \frac{\nu}{\tau_{0}+\tau}, \mu(x, \tau)=\left(\frac{1}{2}+\right.$ $\left.l \frac{d S(x)}{d x}\right) \frac{\nu}{\tau_{0}+\tau}$. With parameters $l=0.1, t=10^{6}$. Chemotactic substance profile, D, scaled for illustration. A: Approximate simulated solution to eq. (6.4) with no nonlinear tempering. B: Simulated results of eq. (6.5) with nonlinear tempering terms $\alpha_{\lambda}(\rho(x, t))=$ $0.9 \rho(x, t), \alpha_{\mu}(\rho(x, t))=0.1 \rho(x, t)$. C: Effect of stronger nonlinear tempering terms to eq. $(6.5) \alpha_{\lambda}(\rho(x, t))=9 \rho(x, t), \alpha_{\mu}(\rho(x, t))=\rho(x, t)$.

\section{References}

[1] E. Barkai, R. Metzler, J. Klafter. From continuous time random walks to the fractional Fokker-Planck equation. Physical Review E, 61 (1) (2000), 132-138.

[2] S. Fedotov. Subdiffusion, chemotaxis, and anomalous aggregation. Physical Review E, 83 (2) (2011), 1-5.

[3] S. Fedotov. Nonlinear subdiffusive fractional equations and the aggregation phenomenon. Physical Review E, 88 (3) (2013), 032104.

[4] S. Fedotov, S. Falconer. Subdiffusive master equation with space-dependent anomalous exponent and structural instability. Physical Review E, 85 (3) (2012), 1-6.

[5] S. Fedotov, S. Falconer. Random death process for the regularization of subdiffusive fractional equations. Physical Review E, 87 (5) (2013), 052139.

[6] S. Fedotov, S. Falconer. Nonlinear degradation-enhanced transport of morphogens performing subdiffusion. Physical Review E, 89 (1) (2014), 012107.

[7] S. Fedotov, A. Ivanov, A.Y. Zubarev. Non-homogeneous random walks, subdiffusive migration of cells and anomalous chemotaxis. Mathematical Modelling of Natural Phenomena, 8 (2) (2013), 28-43.

[8] S. Fedotov, N. Korabel. Subdiffusion in an external potential: Anomalous effects hiding behind normal behavior. Physical Review E, 91 (2015), 042112.

[9] S. Fedotov, V. Méndez. Non-Markovian model for transport and reactions of particles in spiny dendrites. Physical Review Letters, 101 (2008), 218102.

[10] S. Havlin, D. Movshovitz, B. Trus, G.H. Weiss. Probability densities for the displacement of random walks on percolation clusters. Journal of Physics A, 18 (12) (1985), L719.

[11] T. Hillen, K. Painter. Global existence for a parabolic chemotaxis model with prevention of overcrowding. Advances in Applied Mathematics, 26 (4) (2001), 280-301.

[12] T. Hillen, K.J. Painter. A user's guide to pde models for chemotaxis. Journal of Mathematical Biology, 58 (1-2) (2009), 183-217.

[13] S.T. Johnston, M.J. Simpson, R.E. Baker. Mean-field descriptions of collective migration with strong adhesion. Physical Review E, 85 (5) (2012), 051922. 
[14] M.M. Meerschaert, P. Straka. Semi-Markov approach to continuous time random walk limit processes. The Annals of Probability, 42 (4) (2014), 1699-1723.

[15] V. Mendéz, S. Fedotov, W. Horsthemke. Reaction-transport systems: Mesoscopic foundations, fronts, and spatial instabilities. Springer, 2010.

[16] R. Metzler, J. Klafter. The random walk's guide to anomalous diffusion: A fractional dynamics approach. Physics Reports, 339 (1) (2000), 1-77.

[17] R. Metzler, J. Klafter, I.M. Sokolov. Anomalous transport in external fields: Continuous time random walks and fractional diffusion equations extended. Physical Review E, 58 (2) (1998), 1621-1633.

[18] M. Raberto, E. Scalas, F. Mainardi. Waiting-times and returns in high-frequency financial data: an empirical study. Physica A: Statistical Mechanics and its Applications, 314 (2002), 749-755.

[19] M. Rausand, A. Høyland. System reliability theory: Models, statistical methods, and applications. Wiley Series in Probability and Statistics - Applied Probability and Statistics Section. Wiley, 2004.

[20] F. Santamaria, S. Wils, E. De Schutter, G.J. Augustine. The diffusional properties of dendrites depend on the density of dendritic spines. European Journal of Neuroscience, 34 (4) (2011), 561-568.

[21] M.J. Saxton. Anomalous subdiffusion in fluorescence photobleaching recovery: A Monte Carlo study. Biophysical Journal, 81 (4) (2001), 2226-40.

[22] H. Scher, E.N. Montroll. Anomalous transit-time dispersion in amorphous solids. Physical Review B, 12 (6) (1975), 2455-2477.

[23] P. Straka, S. Fedotov. Transport equations for subdiffusion with nonlinear particle interaction. Journal of Theoretical Biology, 366 (2015), 71-83.

[24] M. Vlad, J. Ross. Systematic derivation of reaction-diffusion equations with distributed delays and relations to fractional reaction-diffusion equations and hyperbolic transport equations: Application to the theory of Neolithic transition. Physical Review E, 66 (6) (2002), 1-11.

[25] A. Yadav, W. Horsthemke. Kinetic equations for reaction-subdiffusion systems: Derivation and stability analysis. Physical Review E, 74 (6), 2006. 